Cytogenet Genome Res 1997;78:305-306

\title{
Subject Index, Vol. 78, 1997
}

This index does not contain items of the chromosomes 1,10 and 12 mapping workshop reports. Cytogenetics and Cell Genetics

Addendum

Chromosome 12 mapping report 182

Minireview 55 Animals

Akodon cursor (rodent) 224

Akodon montensís (rodent) 224

Bos grunniens (yak) 69

Bos taurus (cattle) 69

Canisfamiliaris (dog) 231

chicken 124,229

Eulemurfulvus mayottensis (lemur) 260

Euíemur macaco macaco (lemur) 260

Felis catus (domestic cat) 135

gorilla, orangutan 12

Gymnotus carapo (fish) 236

lemur 147,260

mouse 25, 221

Ovis aríes (sheep) 272

Parotomys brantsii (whistling rat) 216

Petrogale (rock wallaby) 74

Presbytis cristata (Old world monkey) 12

Proechimys guairae guairae, $P$. trinüatis

(spiny rats) 36

rat 137

Sorex araneus (shrew) 253

Tursiops truncatus (Atlantic bluenose

dolphin) 6

Banded chromosome analysis A. cursoiiGTG) $224 \operatorname{cat}(\mathrm{Q}) 135 \operatorname{dog}(\mathrm{GTG}) 231 \operatorname{dolphin}(\mathrm{GBG}) 6$ fish(Ag-NOR,C) 236 human(DAPI) 105,289 human(DAPIG) 21 human(G) 58,103,112,247

human(GTG) 96 human(R) 107 human(R,Q) 61 lemur(DAP/G) 260 lemur(R) 147

monkey(DAPI, G) 12 mouse(DAPI) 291 mouse(G) 25 rockwallaby(C) 74 spiny $\operatorname{rat}(C) 36$

whistling $\operatorname{rat}(\mathrm{G}, \mathrm{C}) 216$

Cell cycle

M33 localization 50

Chromosome aberration aneuploidy 27, 116, 120 deletions 240 disomy 116

induction and repair 197 monosomy 96 nondisjunction 27,96 pericentric inversions 12, 224, 236 rearrangements 12,224,260 translocations 202, 247 
Chromosome mapping workshop

chromosome 1153

chromosome 10183

chromosome 1281 Comparative gene mapping

Akodon 224

gorilla 12

lemur 147,260

Old world monkey 12

orangutan 12

rat 137

sheep 272

Erratum 132 Evolution

ancestral chromosomes 12, 137, 224, 260

Bovidae 272

Lemuridae 147,260

Otomyinae 216

telomeric sequences 12,74

Flow cytometry

lemur 260 Fluorescent in situ hybridization (FISH)

breakpoint determination 247

chromosome specific painting probes 124 ,

260

comparative chromosome painting 12,147 ,

216

comparative genomic hybridization 65

direct R banding 107,285,295

interphase FISH 12,27,96

intrachromosomal telomere detection 12 ,

$74,112,224$

multicolor FISH 27,116

NOR localization 6,231

reciprocal chromosome painting 260

Gene mapping cat

MYC 135 chicken

SCD1 229 dog

rDNA 231 dolphin

rDNA 6 human

ACTBP8 46

CD33L 295

CISH 209

endogenous retroviral loci 1

EST00444(D13S308E) 20

FGF12 48

FRA8E 56

GFRA1 289

HERV-K gag loci 1 
HSN-I locus 140

HTR4 133

MAD2L1 63

MEK1, MEK3, MEK4, MEKK5 301

NDUFA2, NDUFA6, NDUFA7,

NDUFB8, NDUFS8 21

PA2G4 31

PCP4 44

PLCD1 58

POLG 281

SCP1 103

SH3D1A 213

TCF17 285

THRSP 131

TNR 145

TP53BP2 61

UBE2E2 107

XPNPEPL 275

YWHAE 105 mouse

Acadvl 25

Gfral 291

Polg 281

Tnr 145 rat

OlßÇ,Rfp 137 Sheep

IGFl,PTHLH,RARG 272 Gene structure

PLCD1 58

TNR 145 Genetic damage

gene amplification 65 repair by exogenous ligase 197 Genome organization spiny rat 36

Heterochromatin

centromere 50

M33 protein 50

Petrogalepenicillata complex 74

Proechimys 36 Human disorders

Alport syndrome 240

breast cancer 105

carcinoma in situ 1

congenital malformations 12

diffuse leiomyomatosis 240

dominant optic atrophy 21,48

Down syndrome 44,213

encephalomyopathies 21

ependymona 247

germ cell tumors 1

hereditary multiple exotosis 56

hereditary sensory neuropathy type I 140

Langer-Giedion syndrome 56 
limb girdle muscular dystrophy 21

lung cancer 58,209

mental retardation 12

myelodysplastic syndrome 63

neurofibromatosis type I 96

nonlymphocytic leukemia 63

ovarian cancer 46

testicular cancer 120

tuberous sclerosis 96

\section{KAIUiEH}

E-Mail karger@karger.ch Fax + 41613061234

(C) 1997 S. Karger AG, Basel

305

Hybrids

human-rodent panel 213

male sterility 69,253

microsatellite instablity 221

radiation hybrid $31,46,301$

rat-mouse 137

somatic cell hybrid panel 1 , 44, 291

Immunofluorescence M33 localization 50

Karyotype

Atlantic bluenose dolphin 6

lemur 260

P. cristata 12

sperm before and after chemotherapy 120

P. brantsii 216

Linkage

HSN-I locus on 9q22 140

Meiosis

common shrew 253

Fl hybrids and backcross progeny 69

segregation patterns of reciprocal transloca-

tions 202

synaptonemal complex protein 1103 Microdissection and microcloning

A. cursor 224

chicken $\mathrm{Z}$ chromosomes 124

NOR analysis dog 231 dolphin 6 fish 236 shrew 253

Physical mapping

FRA8E region at 8q24.11 56 interstitial telomere sites 112 PLCDlat3p21.3 58 subtelomere YAC panel 12

Repeats

centromeric sequences 112

microsatellite instability 221

polymorphic pentanucleotide 56 
telomeric sequence 74,112 Restriction mapping COL4A6 240

Sequence

CD33L 295

CISH 209

HERV-Kgag 1

PA2G4 31

POLG 281

repetitive DNA families 36

TCF17 285

UBE2E2 107

XPNPEPL 275 Sex chromosomes

aneuploidy in sperm 27, 116, 120

chicken 124

paternal age effect on aneuploidy 116

Techniques

aneuploidy in human sperm pronuclei 27

electroporation 197

limits of detection of copy number in $\mathrm{CGH}$

65

PRINS dual color in sperm pronuclei 202

PTT for intact reading frame detection 1

306

Cytogenet Cell Genet Vol. 78, 1997 